Arthritis Care \& Research

Vol. 62, No. 1, January 15, 2010, pp 137-142

(C) 2010, American College of Rheumatology

\title{
LETTERS
}

DOI 10.1002/acr.20025

\section{Value of assessing the fifth metatarsophalangeal joint in patients with early rheumatoid arthritis: comment on the article by Sheane et al}

\section{To the Editors:}

We read with interest the article published in Arthritis Care $\&$ Research by Sheane et al on the value of examining by ultrasonography (US) the fifth metatarsophalangeal (MTP) joint for the early diagnosis of rheumatoid arthritis (RA) (1). We fully agree with Sheane et al on the value of examining this joint in patients suspected of having RA. We were, however, surprised that our previously published work was not included on their reference list. Our work indicates that erosive disease was most commonly seen in the fifth MTP of all joints (2). US of the feet revealed erosive changes in 3 patients who had negative US of their hands. One of these patients had no evidence of erosive disease in any of the other joints of the hands or feet usually assessed by radiography. The table included in our previous publication provides the specific numbers.

Graciela S. Alarcón, MD, MPH

Robert Lopez-Ben, MD

University of Alabama at Birmingham

1. Sheane BJ, Beddy P, O’Connor M, Miller S, Cunnane G. Targeted ultrasound of the fifth metatarsophalangeal joint in an early inflammatory arthritis cohort. Arthritis Rheum 2009;61: 1004-8.

2. Lopez-Ben R, Bernreuter WK, Moreland LW, Alarcon GS. Ultrasound detection of bone erosions in rheumatoid arthritis: a comparison to routine radiographs of the hands and feet. Skeletal Radiol 2004;33:80-4.
DOI 10.1002/acr.20026

\section{Ultrasound of metatarsophalangeal joints in an early inflammatory arthritis cohort: comment on the article by Sheane et al}

\section{To the Editors:}

We read with great interest the recent article published in Arthritis Care $\&$ Research by Sheane et al about the role of ultrasonography (US) in the evaluation of the fifth metatarsophalangeal (MTP) joint in patients with early arthritis (EA) (1). In the assessment of patients with EA, it is very important to distinguish between different forms of arthritides, such as rheumatoid arthritis (RA) and undifferentiated arthritis (UA), as early as possible after symptom development (2). Recently, US has been indicated as being more sensitive than clinical examination and conventional radiography in the detection of joint erosive damage, and it is frequently applied in the assessment of patients affected by EA (3). Sheane et al, who evaluated 30 patients with a new diagnosis of inflammatory arthritis, indicated that US examination of the fifth MTP joint aids in the identification of early erosive disease and consequent diagnosis of RA. Erosions, synovial hypertrophy, and power Doppler (PD) signal were evaluated, detecting the evidence of fifth MTP joint erosions in more than $50 \%$ of patients, which was significantly higher in those with a diagnosis of early RA compared with UA. Significant differences between radiographic- and US-detected erosions in the fifth MTP joint were evident, with correlation between US-detected synovial hypertrophy and radiographic erosions. We would like to address the following comments.

First, Sheane and colleagues seem to consider the presence of synovial hypertrophy as indicative of synovitis and use those 2 terms as being synonymous. We wonder if this is correct, considering that Outcome Measures in Rheumatology Clinical Trials (OMERACT) definitions refer to synovial hypertrophy and synovial fluid and do not mention the term "synovitis." Second, we believe that the use of warm water bath could influence the appearance

\begin{tabular}{|lcccc|}
\hline \multicolumn{5}{|c|}{ Table 1. US findings in 49 patients with early RA and UA* } \\
\hline & $\begin{array}{c}\text { RA patients } \\
(\mathbf{n}=\mathbf{2 5})\end{array}$ & $\begin{array}{c}\text { UA patients } \\
(\mathbf{n}=\mathbf{2 4})\end{array}$ & $\begin{array}{c}\text { Total } \\
\text { patients } \\
(\mathbf{n}=\mathbf{4 9})\end{array}$ & $\boldsymbol{P}$ \\
\hline US erosions fifth MTP joint & $3(12)$ & $1(4)$ & $4(8.1)$ & NS \\
US synovial proliferation fifth MTP joint & $3(12)$ & $2(8.3)$ & $5(10.2)$ & NS \\
US synovial effusion fifth MTP joint & $6(25)$ & $3(12.5)$ & $9(18.3)$ & NS \\
PD-positive fifth MTP joint & $2(8)$ & $1(4)$ & $3(6)$ & NS \\
\hline * Values are the number (percentage) unless otherwise indicated. All comparisons of frequency between \\
the groups were made using Fisher's exact test. All P values were 2-tailed, and the significance cutoff was \\
$P$ < 0.05. US = ultrasonography; RA = rheumatoid arthritis; UA = undifferentiated arthritis; MTP = \\
metatarsophalangeal; NS = not significant; PD = power Doppler. \\
\hline
\end{tabular}




\begin{tabular}{|c|c|c|c|c|}
\hline & $\begin{array}{l}\text { RA MTP joints } \\
\quad(n=250)\end{array}$ & $\begin{array}{l}\text { UA MTP joints } \\
\quad(n=240)\end{array}$ & $\begin{array}{l}\text { Total joints } \\
(n=490)\end{array}$ & $\boldsymbol{P}$ \\
\hline US erosions MTP joints & $32(12.8)$ & $14(5.8)$ & $46(9.3)$ & 0.008 \\
\hline US synovial proliferation MTP joints & $53(21.2)$ & $11(4.6)$ & $64(13.1)$ & $<0.001$ \\
\hline US synovial effusion MTP joints & $66(26.4)$ & $36(15)$ & $102(20.1)$ & 0.002 \\
\hline PD-positive MTP joints & $10(4)$ & $8(3.3)$ & $18(3.7)$ & NS \\
\hline \multicolumn{5}{|c|}{$\begin{array}{l}\text { * Values are the number (percentage) unless otherwise indicated. All comparisons of frequency between the } \\
\text { groups were made using Fisher's exact test. All } P \text { values were 2-tailed, and the significance cutoff was } P<0.05 \text {. } \\
\text { US = ultrasonography; MTP = metatarsophalangeal; RA = rheumatoid arthritis; UA = undifferentiated arthritis; } \\
\text { PD = power Doppler; NS = not significant. }\end{array}$} \\
\hline
\end{tabular}

of PD signal, due to vasodilatation correlated to increase of local temperature. The use of US gel and the avoidance of probe pressure over the area of interest are commonly considered as reliable procedures for correct sonographic scanning of musculoskeletal structures and usually ensure a good image quality (4). Furthermore, after reading this interesting study by Sheane et al, we would like to report our experience with 49 consecutive patients with EA who were referred to the EA clinic of the rheumatology unit at Sapienza University of Rome. In our cohort, we examined MTP joints both by grey scale and PD US by using a Philips/HP Image Point HX machine (Philips, Amsterdam, The Netherlands) equipped with a $14 \mathrm{MHz}$ linear probe and PD (setting: frequency $7 \mathrm{MHz}$, pulse repetition frequency $700-1,000$, gain $18-30 \mathrm{~dB}$, low filter). US gel was used. According to OMERACT definitions, we evaluated synovial fluid, synovial proliferation, PD, and erosions (5). All findings were scored by using a dichotomous assessment $(0=$ absent, $1=$ present). US findings in MTP joints of patients with EA, subgrouped according to a diagnosis of either RA or UA, are reported in Tables 1 and 2, where US modifications referred only to the fifth MTP joint and all MTP joints are described.

In our cohort, US evaluation of the fifth MTP joint showed a lower percentage of patients with bony erosions compared with the study by Sheane et al $(8.1 \%$ versus $56.7 \%$ ), confirming a higher prevalence in RA patients with respect to UA ( $12 \%$ versus $4 \%$ ). Moreover, we did not find significant differences between the 2 groups of patients concerning the presence of PD signal. The extension of US evaluation to bilateral first to fifth MTP joints in all 49 patients confirmed a similar prevalence of erosions detected by US (9.3\% versus $8.1 \%)$. By a different point of view, based on the analysis of MTP joint involvement, we demonstrated significant differences between RA and UA concerning the percentage of joints with erosions, synovial effusion, and proliferation $(P=0.008, P<0.001$, and $P=$ 0.002 , respectively).

In conclusion, according to our experience, we agree with Sheane and colleagues on the relevant role of US of the fifth MTP joint in the assessment of patients with EA. Considering, however, that all MTP joints were more frequently and earlier involved in RA than in UA, we propose the extension of US evaluation to all of the MTP joints and not only to the fifth. In our opinion, this procedure would not significantly increase the time duration of a single US examination session and would improve the entity of in- formation obtained, thereby helping to differentiate between early RA and UA. Further studies on larger cohorts and longitudinal research studies are needed to confirm these results.

Fulvia Ceccarelli, MD

Annamaria Iagnocco, MD

Manuela Di Franco, MD

Cristina Iannuccelli, MD

Guido Valesini, MD

Sapienza University of Rome

Rome, Italy

1. Sheane BJ, Beddy P, O’Connor M, Miller S, Cunnane G. Targeted ultrasound of the fifth metatarsophalangeal joint in an early inflammatory arthritis cohort. Arthritis Rheum 2009;61: 1004-8.

2. Visser H, le Cessie S, Vos K, Breedveld FC, Hazes JM. How to diagnose rheumatoid arthritis early: a prediction model for persistent (erosive) arthritis. Arthritis Rheum 2002;46:357-65.

3. Szkudlarek M, Narvestad E, Klarlund M, Court-Payen M, Thomsen HS, Ostergaard M. Ultrasonography of the metatarsophalangeal joints in rheumatoid arthritis: comparison with magnetic resonance imaging, conventional radiography, and clinical examination. Arthritis Rheum 2004;50:2103-12.

4. Iagnocco A, Epis O, Delle Sedie A, Meenagh G, Filippucci E, Riente L, et al. Ultrasound imaging for the rheumatologist. XVII. Role of colour Doppler and power Doppler. Clin Exp Rheumatol 2008;26:759-62.

5. Wakefield RJ, Balint PV, Szkudlarek M, Filippucci E, Backhaus M, D’Agostino MA, et al. OMERACT 7 Special Interest Group: musculoskeletal ultrasound including definitions for ultrasonographic pathology. J Rheumatol 2005;32:2485-7.

DOI 10.1002/acr.20027

Reply

\section{To the Editors:}

We thank Drs. Alarcón, Lopez-Ben, and Ceccarelli et al for their interest in our work, and we welcome both their comments and research findings in relation to the use of US in the setting of early inflammatory arthritis.

Ceccarelli et al note the possible contrast in definition between the terms "synovitis" and "synovial hypertrophy." Histologically, both names relate to a pathologic process within the joint, resulting from current or previous inflammatory change within the synovium. We used the word synovitis according to the US description outlined by Szkudlarek et al, who defined it as "a non- 